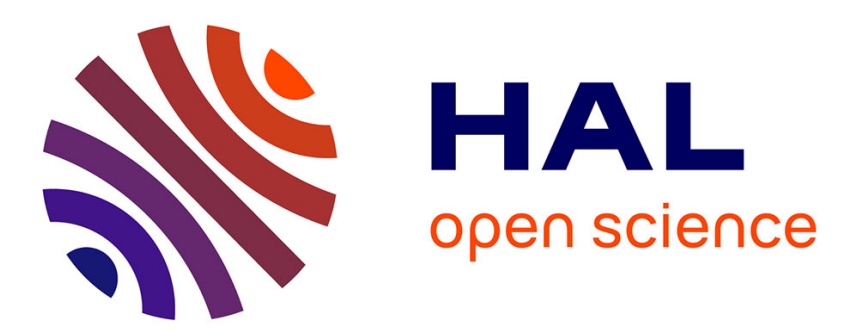

\title{
Emission Spectroscopy of the Cathode Fall Region of an Analytical Glow Discharge
}

\author{
N. Konjevic, I. Videnovic, M. Kuraica
}

\section{To cite this version:}

N. Konjevic, I. Videnovic, M. Kuraica. Emission Spectroscopy of the Cathode Fall Region of an Analytical Glow Discharge. Journal de Physique IV Proceedings, 1997, 07 (C4), pp.C5-247-C5-258. 10.1051/.jp4:1997420 . jpa-00255576

\section{HAL Id: jpa-00255576 https://hal.science/jpa-00255576}

Submitted on 1 Jan 1997

HAL is a multi-disciplinary open access archive for the deposit and dissemination of scientific research documents, whether they are published or not. The documents may come from teaching and research institutions in France or abroad, or from public or private research centers.
L'archive ouverte pluridisciplinaire HAL, est destinée au dépôt et à la diffusion de documents scientifiques de niveau recherche, publiés ou non, émanant des établissements d'enseignement et de recherche français ou étrangers, des laboratoires publics ou privés. 


\title{
Emission Spectroscopy of the Cathode Fall Region of an Analytical Glow Discharge
}

\author{
N. Konjević, I.R. Videnović and M.M. Kuraica
}

Faculty of Physics, University of Belgrade, P.O. Box 368, 11001 Belgrade, Yugoslavia

\begin{abstract}
Stark spectroscopy of hydrogen Balmer lines and neutral helium lines with forbidden components is used for the study of an analytical glow discharge.

Splitting of the hydrogen $\mathrm{H}_{\beta}$ and $\mathrm{H}_{\gamma}$ lines is applied for simultaneous determination of local electric field and temperature of excited $\mathrm{H}$ atoms in pure hydrogen and in argon-hydrogen mixture. Temperature measuremients showed that, in pure hydrogen discharge, at least two groups of excited atoms exist: "slow", with average energies in the range from $3.4 \mathrm{eV}$ to $8.2 \mathrm{eV}$ and "fast", ranging between $80 \mathrm{eV}$ and $190 \mathrm{eV}$. In argon-hydrogen discharge, excited hydrogen neutrals with average energies between $32 \mathrm{eV}$ and $43 \mathrm{eV}$ are detected only. The origin of these energetic neutrals is related to the presence of $\mathrm{H}^{+}$and $\mathrm{H}_{3}{ }^{+}$ions in pure hydrogen, and to the dominant role of $\mathrm{H}_{3}{ }^{+}$ion in argon-hydrogen mixture. For both gases, in the negative glow region, the increase of the exited hydrogen atoms temperature is detected and explanation suggested.

For the electric field measurements in pure helium and helium-hydrogen mixture, the Stark splitting and shifting of the neutral helium $492.19 \mathrm{~nm}, 447.15 \mathrm{~nm}$ and $406.62 \mathrm{~nm}$ with respect to their forbidden counterparts is used. Good agreement between electric field measurements from these three helium lines is obtained.
\end{abstract}

\section{INTRODUCTION}

A glow discharge source (GDS) with a flat cathode and cylindrical hollow anode, first described by Grimm [1], is a well established excitation source for the analysis of conducting solid samples by optical emission spectroscopy [2,3], see also [4]. Owing to sample ablation by cathodic sputtering, matrix effects resulting from selective volatilization are absent. Because of the layer by layer ablation of the sample surface, the Grimm GDS is used for in-depth profiling analysis [5,6]. Similar advantages are realized when atomic absorption spectrometry is performed with a Grimm GDS [7,8]. In the last several years various types of GDSs were also used as ion sources for mass spectrometry [9]. For solids analysis, direct current (DC) GDSs have been successfully complemented by the radio-frequency (RF) discharges. Namely, the use of DC discharges is limited to metals, whereas the RF discharges are applicable for nonconducting materials also (see [10] and references therein). A comprehensive list of publications dealing with GDSs and their applications is given in [11-13].

However, if one scans the literature dealing with Grimm GDS, see e.g. [11,12], it is evident that most of the papers are devoted to various applications. Only few report results of the discharge parameter measurements and they are mainly concentrated on volt-ampere characteristics, cathode surface studies and measurements of relative line intensities for different discharge conditions. This observation may be generalized to all analytic. GDSs. In order to supply new reliable data, several years ago, we set up in our laboratory an experiment to measure plasma parameters of a modified Grimm GDS. First results of negative glow plasma diagnostics (electron density and temperature, excitation temperatures and gas temperature) are obtained in argon with a small admixture (3\%) of hydrogen [14]. Unexpected intensive spectral line broadening of the wings of all hydrogen Balmer lines was detected and studied in detail $[15,16]$.

In this paper we resume results of the experimental studies [17-19] of the cathode fall region (CFR) of the Grimm GDS. The study of the physical processes in this part of the discharge is of importance for understanding the operation of the GDS and the sputtering processes at the cathode surface which are both 
of primary interest for analytical applications. Here we use Stark spectroscopy of hydrogen Balmer lines for spatial distribution measurements of the electric field intensity and the temperature of excited hydrogen atoms in the CFR of pure hydrogen and argon-hydrogen mixture discharges. In pure helium and helium-hydrogen mixture, we applied Stark splitting and shifting of the neutral helium $492.19 \mathrm{~nm}\left(2 p^{1} \mathrm{P}^{0}\right.$ $\left.-4 d^{1} \mathrm{D}\right), 447.15 \mathrm{~nm}\left(2 p^{3} \mathrm{P}^{0}-4 d^{3} \mathrm{D}\right)$ and $402.62 \mathrm{~nm}\left(2 p^{3} \mathrm{P}^{0}-5 d^{3} \mathrm{D}\right)$ lines with respect to their forbidden counterparts $\left(2 p^{1} \mathrm{P}^{0}-4 f^{1} \mathrm{~F}\right),\left(2 p^{3} \mathrm{P}^{0}-4 f^{3} \mathrm{~F}^{0}\right)$ and $\left(2 p^{3} \mathrm{P}^{0}-5 f^{3} \mathrm{~F}^{0}\right)$, respectively, for electric field spatial distribution measurements in the cathode fall region of the Grimm GDS.

\section{EXPERIMENTAL APPARATUS AND PROCEDURE}

Since we describe here two sets of measurements, one in pure hydrogen and in an argon-hydrogen mixture, and the other one in pure helium and in a helium-hydrogen mixture, common details of both experiments will be outlined first. Their specific details will be given separately. The latter experiment, in pure helium and in the helium-hydrogen mixture, is considerably improved and therefore its setup is schematically presented in Fig. 1.

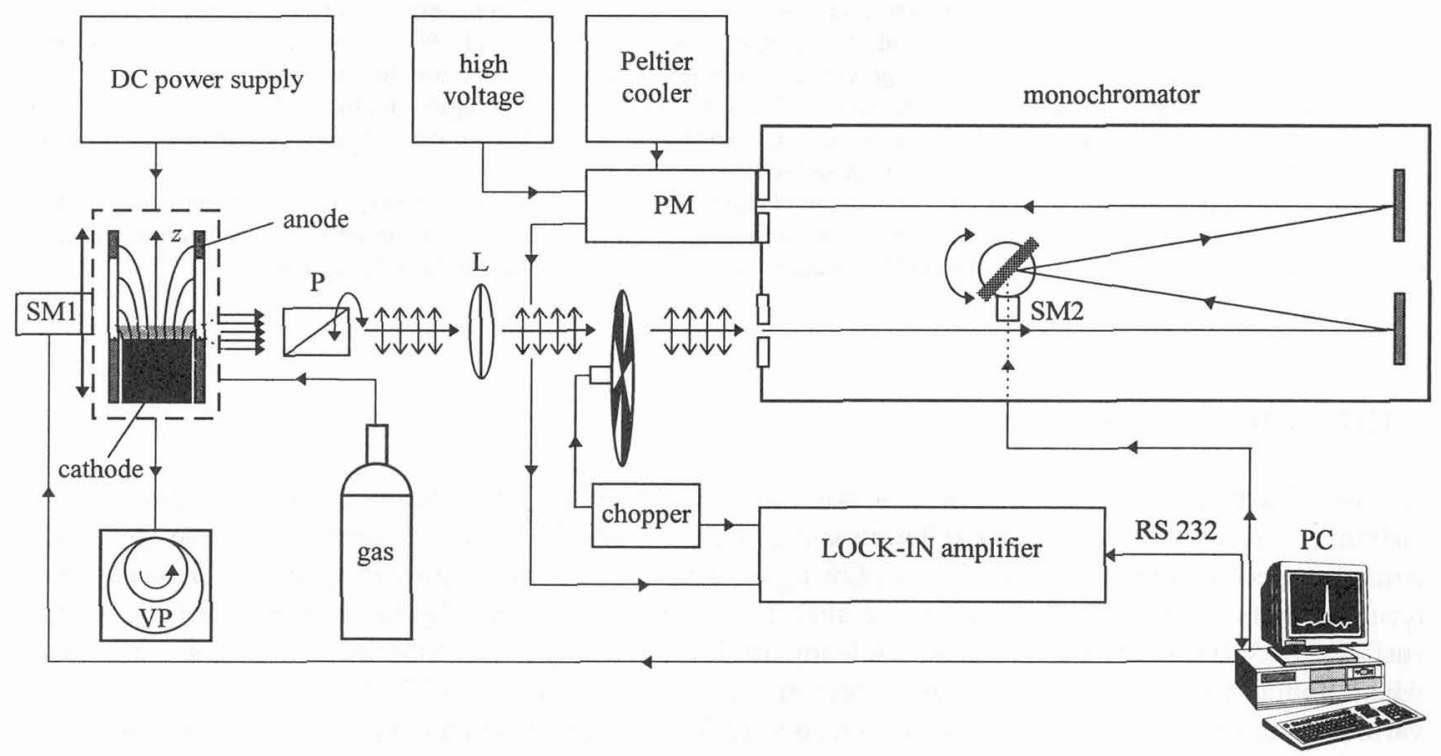

Fig. 1. Schematic diagram of the central part of the Grimm GDS and experimental setup for side-on observations in the heliumbased experiments. Symbols: VP - vacuum pump, SM - stepping motors; P - polarizer; L - lens; PM - photomultiplier.

\subsection{Common details}

Our discharge source, a modified Grimm GDS, is laboratory made after Ferreira et al. [20] and described in detail elsewhere [14]. Here, for completeness, only minimum information will be given. The hollow anode, $30 \mathrm{~mm}$ long with inner and outside diameters 8.00 and $13 \mathrm{~mm}$, has a longitudinal slot (15 mm long and $1 \mathrm{~mm}$ wide) for side-on observations along the discharge axis. The water cooled cathode holder has an exchangeable iron electrode, $18 \mathrm{~mm}$ long and $7.60 \mathrm{~mm}$ in diameter, which screws tightly onto its holder to ensure good cooling. Unlike the original Grimm design, the cathode is mounted inside the hollow anode. A gas flow was sustained at a selected pressure by means of a needle valve and a two-stage mechanical vacuum pump. In order to prevent backstreaming of oil vapors, the zeolite trap is mounted between discharge vessel and vacuum pump. To run the discharge, a $0-2 \mathrm{kV}$ and $0-100 \mathrm{~mA}$ current stabilized power supply (Kepco BHK $2000-01 \mathrm{M}$ ) is used. A ballast resistor of $10 \mathrm{k} \Omega$ is placed in series with the discharge and power supply. 
After polarization with the Glan-Thomson prism or plastic polarizer, the radiation from the discharge source is focused with unity magnification onto the entrance slit of the scanning monochromatorphotomultiplier system, see Fig. 1. For electric field intensity axial distribution measurements, the discharge tube is moved in about $0.1 \mathrm{~mm}$ steps along the discharge axis, so the discharge image obtained through the observation slot is shifted in the plane of the entrance slit of the monochromator, see Fig. 1. The photomultiplier signals were A/D converted, collected and processed by a PC.

\subsection{Specific details}

\subsubsection{Experiment in pure hydrogen and in the argon-hydrogen mixture $\left(97 \% \mathrm{Ar}: 3 \% \mathrm{H}_{2}\right)$ :}

gas flow:

pressure:

spatial resolution:

monochromator:

slit width:

$300-500 \mathrm{~cm}^{3} / \mathrm{min}$ (at room temperature and atmospheric pressure);

195-250 $\mathrm{Pa}$ (pure $\mathrm{H}_{2}$ ) and 240-425 $\mathrm{Pa}\left(\mathrm{Ar}-\mathrm{H}_{2}\right)$;

$0.02 \mathrm{~mm}$ (pure $\mathrm{H}_{2}$ ) and $0.01 \mathrm{~mm}\left(\mathrm{Ar}-\mathrm{H}_{2}\right)$, along the discharge axis;

$2 \mathrm{~m}$ PGS-2 Carl Zeiss Jena (Ebert type), $640 \mathrm{~mm}^{-1}$ reflection grating, 0.74

$\mathrm{nm} / \mathrm{mm}$ inverse linear dispersion in the first order;

same entrance and exit slits of $15 \mu \mathrm{m}$ and $30 \mu \mathrm{m}$ for the $\mathrm{H}_{\beta}$ and $\mathrm{H}_{\gamma}$ spectra recordings, respectively, and $15 \mu \mathrm{m}$ entrance and $50 \mu \mathrm{m}$ exit slits for the integral $\mathrm{Fe} I, \mathrm{Ar} I$ and Ar II line intensities measurements;

instrumental halfwidth: $0.014 \mathrm{~nm}$ with $15 \mu \mathrm{m}$ entrance and exit slits (Gaussian form);

radiation detection: photomultiplier EMI $9789 \mathrm{QB}$.

\subsubsection{Experiment in pure helium (99.95\%) and in the helium-hydrogen mixture (95\% $\left.\mathrm{He}: 5 \% \mathrm{H}_{2}\right)$ :}

gas flow:

pressure:

spatial resolution:

monochromator:

slit width: instrumental halfwidth: $0.022 \mathrm{~nm}$ (Gaussian form);

radiation detection: Peltier-cooled photomultiplier, lock-in amplifier (SR 510 Stanford Research) with radiation chopper (SR 540 Stanford Research) operating at $83.3 \mathrm{~Hz}$.

\section{THEORY}

\subsection{Theoretical basis for electric field and excited hydrogen temperature measurements using Stark splitting of hydrogen Balmer lines}

The splitting of energy levels of the hydrogen and hydrogen-like emitter in an external electric field is successfully described both by semiclassical and quantum mechanical theory of the linear Stark effect (see e.g. $[21,22]$ ). Both theories yield the same result: an energy level with principal quantum number $n$ is splitted into $2 n-1$ equidistant sub-levels determined by quantum numbers $k(|k|<n)$. Therefore, the spectral line emitted as a transition between energy levels 1 and 2 of the hydrogen atom consists of numerous components which are polarized either linearly, parallel to the vector of external field $E(\Delta \mathrm{m}=$ 0 , or $\pi$-components), or circularly, in the plane perpendicular to $E$ ( $\Delta \mathrm{m}= \pm 1$, or $\sigma$-components). Stark components are wavelength shifted from the line center by the value

$$
\left(\mathrm{n}_{1} \mathrm{k}_{1}-\mathrm{n}_{2} \mathrm{k}_{2}\right) \Delta \lambda_{0} \text {, }
$$

where $\Delta \lambda_{0}$ is the smallest shift determined by the local field intensity [22]: 


$$
\begin{aligned}
& \Delta \lambda_{0}[\mathrm{~nm}]=1.52 \cdot 10^{-3} E[\mathrm{kV} / \mathrm{cm}]-\text { for the } \mathrm{H}_{\beta} \text { line, } \\
& \Delta \lambda_{0}[\mathrm{~nm}]=1.21 \cdot 10^{-3} E[\mathrm{kV} / \mathrm{cm}]-\text { for the } \mathrm{H}_{\gamma} \text { line. }
\end{aligned}
$$

With the polarizer axis set parallel or perpendicular to the electric field, the appropriate ( $\pi$ or $\sigma$ ) overall profile is formed. In order to create overall $\pi$ and $\sigma$ Stark profiles we assumed that plasma broadening effects in the cathode fall region may be neglected; calculations of Bogaerts et al. [23] show that electron densities in the cathode dark space of an analytical glow discharge at $1000 \mathrm{~V}$ and $100 \mathrm{~Pa}$ do not exceed $10^{7} \mathrm{~cm}^{-3}$. Thus, to each Stark component we have assigned a Gauss function only. The full halfwidth $\Delta \lambda_{G}$ of each Gaussian results from Doppler, $\Delta \lambda_{D}$, and instrumental, $\Delta \lambda_{I}$, halfwidths:

$$
\Delta \lambda_{G}=\sqrt{\Delta \lambda_{D}^{2}+\Delta \lambda_{I}^{2}} .
$$

For hydrogen, the Doppler halfwidth can be calculated from

$$
\Delta \lambda_{D}=7.16 \cdot 10^{-7} \lambda_{0} \sqrt{T},
$$

where $\lambda_{0}$ is the central wavelength of the line and $T$ is the temperature of the excited $\mathrm{H}$ atoms. The overall profile is calculated as the superposition of all components:

$$
I(\lambda, E, T)=\sum_{i=1}^{N} I_{0 i} \exp \left\{-\left[\frac{\lambda-\left(\mathbf{n}_{1} \mathbf{k}_{1}-\mathbf{n}_{2} \mathrm{k}_{2}\right)_{i} \Delta \lambda_{0}}{\Delta \lambda_{G} / 2 \sqrt{\ln 2}}\right]^{2}\right\},
$$

where $I_{0 i}$ are relative intensities [21] and $N$ is the total number of components (10 - for the both $\mathrm{H}_{\beta} \pi$ and $\sigma$ profiles, 14 and 13 - for the $\mathrm{H}_{\gamma} \pi$ and $\sigma$ profiles, respectively). The theoretical profiles (5) are fitted to the experimental recordings, by varying the electric field intensity $E$ and the temperature $T$ of excited hydrogen atoms. In the negative glow region, the difference between $\pi$ and $\sigma$ profiles disappears $(E \approx 0)$, and the fitting procedure is reduced to variation of $T$ only. A detailed explanation of the theoretical basis of these measurements, fitting procedure and discussion of the influence of the fine structure splitting on the shape of $\pi$ and $\sigma$ profiles one can find in [17].

\subsection{Theoretical basis for electric field measurements from Stark splitting and shifting of helium lines and their forbidden components}

To measure electric field strengths in the CFR of pure helium and helium-hydrogen mixture discharges, we used the perturbation theory for the evaluation of the displacements of helium energy sub-levels in the external electric field, see e.g. [22]. Following the classical work by Foster [24], we calculated the displacements of the Stark sub-levels of the He I $492.19 \mathrm{~nm}\left(2 p^{1} \mathrm{P}^{0}-4 d^{1} \mathrm{D}\right), 447.15 \mathrm{~nm}\left(2 p^{3} \mathrm{P}^{0}-4 d^{3} \mathrm{D}\right)$ and $402.62 \mathrm{~nm}\left(2 p^{3} \mathrm{P}^{0}-5 d^{3} \mathrm{D}\right)$ lines and their forbidden components $\left(2 p^{1} \mathrm{P}^{0}-4 f^{1} \mathrm{~F}\right),\left(2 p^{3} \mathrm{P}^{0}-4 f^{3} \mathrm{~F}^{0}\right)$ and $\left(2 p^{3} \mathrm{P}^{0}-5 f^{3} \mathrm{~F}^{0}\right)$, respectively, in the electric field ranging from 0 to $20 \mathrm{kV} / \mathrm{cm}$. For these calculations, helium energy levels are taken from Martin [25]. As an example, the results for the $447.15 \mathrm{~nm}$ line (in wavelength units) are given in Fig. 2. For further analysis we concentrate here to the $\pi(\Delta \mathrm{m}=0)$ components which are usually stronger and easier to measure than $\sigma(\Delta \mathrm{m}= \pm 1)$ components. The mutual wavelength separation $\Delta \lambda_{\mathrm{AF}}$ of $\pi$ components of allowed and forbidden line is calculated in $1 \mathrm{kV}$ steps and the results (see Fig. 3) are fitted by polynomial best fits with correlation factors better than 0.9999 . Here, for an electric field ranging from 0 to $20 \mathrm{kV} / \mathrm{cm}$, we neglected splitting of the common lower energy level for all three lines and their forbidden components. From Figs. 2 and 3 (see data for the $402.62 \mathrm{~nm}$ line) it is clear that, within the investigated range of electric field strengths, Stark components arising from transitions between sub-levels with magnetic quantum numbers $m=0$ and $m=1$ of both allowed and forbidden lines are practically unresolvable with our spectral resolution. Therefore with the polarizer mounted with its axis parallel to the external field only one allowed and one forbidden $\pi$ component are detected. Thus, to evaluate the separation $\Delta \lambda_{A F}$ (in $\mathrm{nm}$ ) between corresponding lines we have taken an average value for the $\mathrm{m}_{\text {upper }}=0 \rightarrow \mathrm{m}_{\text {lower }}=0$ and $\mathrm{m}_{\text {upper }}=1 \rightarrow \mathrm{m}_{\text {lower }}=1$ displacements. The obtained results are fitted with following polynomials (see Fig. 3):

$3^{\text {rd }}$ order for the He I $492.19 \mathrm{~nm}$ line:

$$
\Delta \lambda_{\mathrm{AF}}=-1.87(9) \cdot 10^{-5} \cdot E^{3}+8.8(3) \cdot 10^{-4} \cdot E^{2}+1.4(2) \cdot 10^{-3} \cdot E+0.1316(5),
$$




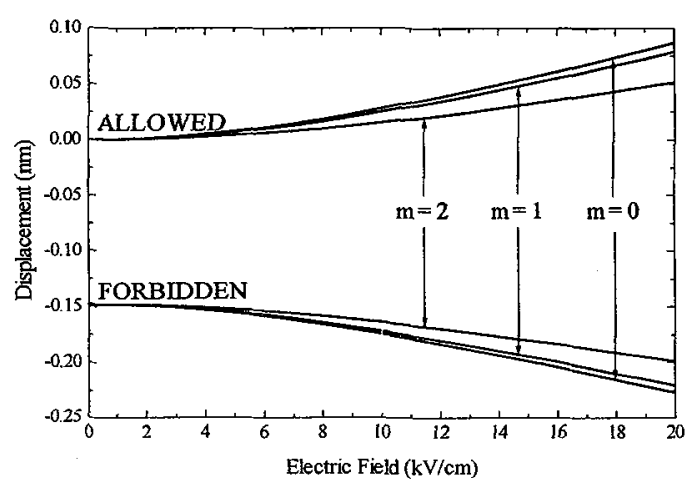

Fig. 2. The calculated sub-level displacements (in wavelength units) of $4 d^{3} \mathrm{D}$ and $4 f^{3} \mathrm{~F}$ helium energy levels, which are the upper levels of the $\mathrm{He} I 447.15 \mathrm{~nm}$ allowed and its forbidden line, respectively.

average values (solid lines, Eqs. (6) and (7) respectively) are given. The reslts (scatered graphs) and $447.15 \mathrm{~m}$ lines only Eqs. (6-8), obtained by measuring $\Delta \lambda_{\mathrm{AF}}$ for all three He I lines vs. electric field strength determined from the $\pi$ shape of $\mathrm{H}_{\beta}$ profile in helium-hydrogen mixture, are also given.

$3^{\text {rd }}$ order for the He I $447.15 \mathrm{~nm}$ line:

$$
\Delta \lambda_{\mathrm{AF}}=-1.06(3) \cdot 10^{-5} \cdot E^{3}+5.95(8) \cdot 10^{-4} \cdot E^{2}+2.5(7) \cdot 10^{-4} \cdot E+0.1479(2),
$$

$4^{\text {th }}$ order for the He I $402.62 \mathrm{~nm}$ line:

$$
\Delta \lambda_{\mathrm{AF}}=1.9(2) \cdot 10^{-6} \cdot E^{4}-9.8(7) \cdot 10^{-5} \cdot E^{3}+1.86(9) \cdot 10^{-3} \cdot E^{2}-2.6(4) \cdot 10^{-3} \cdot E+0.0638(6),
$$

where $E$ is the electric field strength in $\mathrm{kV} / \mathrm{cm}$. In addition, this averaging can be justified by the fact that $\mathrm{m}_{\text {upper }}=0 \rightarrow \mathrm{m}_{\text {lower }}=0$ and $\mathrm{m}_{\text {upper }}=1 \rightarrow \mathrm{m}_{\text {lower }}=1$ components of both allowed and forbidden lines are approximately of the same intensity [24].

In order to test Eqs. (6-8), we performed an experiment in a helium-hydrogen mixture where electric field strengths are independently determined from the shape of $\mathrm{H}_{\beta}(\pi)$ profile (see Eq. (14) in [17]) at various positions along the CFR of our discharge. At the same positions (same electric field strengths) $\Delta \lambda_{\mathrm{AF}}$ for the He I lines are determined and the results are given in Fig. 3. If one takes into account errors of both sets of measurements, the agreement with Eqs. (6-8) is very good.

\section{RESULTS AND DISCUSSION}

\subsection{Pure hydrogen discharge}

The examples of experimental recordings, best fits and the results of electric field intensities at various distances from the cathode derived from the $\mathrm{H}_{\beta}$ and $\mathrm{H}_{\gamma}$ lines are given in Figs. 4 and 5, respectively. Here it should be pointed out that, in the pure hydrogen discharge, best fits of experimental profiles are obtained assuming that two groups of excited hydrogen atoms exist: one with temperatures around $5 \mathrm{eV}$ (so called "slow" neutrals) and another group with temperatures around $100 \mathrm{eV}$ ("fast" neutrals). In the negative glow region, a third central narrow profile is observed, with a width determined by the Stark and Doppler $\left(T_{g} \approx 1000 \mathrm{~K}\right.$, see [14]) effects in the plasma as well as instrumental broadening [14], see Figs. 4 and 5 . It is important to mention also, that by fitting a single experimental profile with a theoretical one (5), results of local electric field, temperatures of "slow" $T_{s}$ and "fast" $T_{f}$ excited hydrogen atoms and their relative concentrations are obtained simultaneously, see [17].

The comparison of experimentally determined electric fields from $\pi$ and $\sigma$ profiles, see Figs. 4 and 5 , shows systematic discrepancies, with the field measured from $\sigma$ being always smaller. These discrepancies have been discussed in detail in [17]. To determine the electric field intensity distribution in 

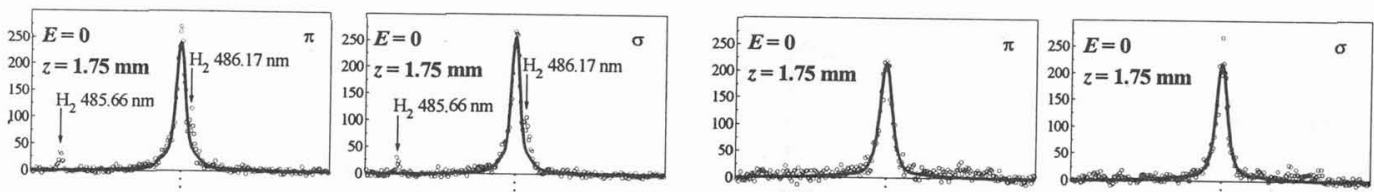

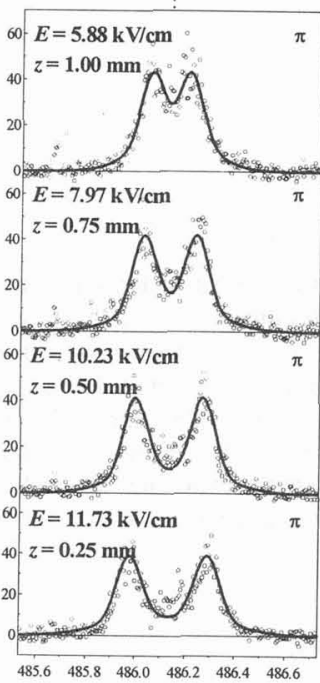

$\lambda(\mathrm{nm})$

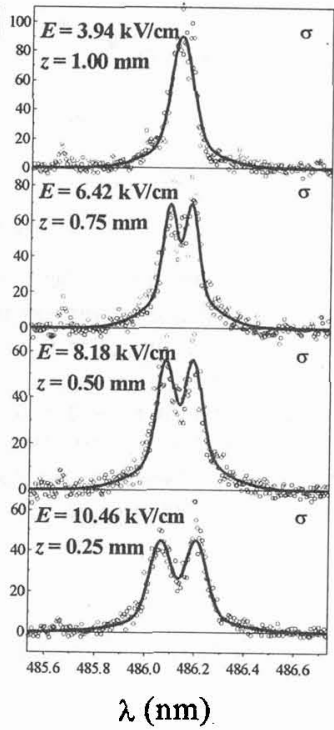

$\lambda(\mathrm{nm})$

Fig. 4. Measured Balmer $\mathrm{H}_{\beta}$ Stark profiles and their best fits (solid lines) at several distances from the cathode. Intensities are given in arbitrary units. Discharge conditions: pure hydrogen at $228 \mathrm{~Pa}, 30 \mathrm{~mA}, 920 \mathrm{~V}$.
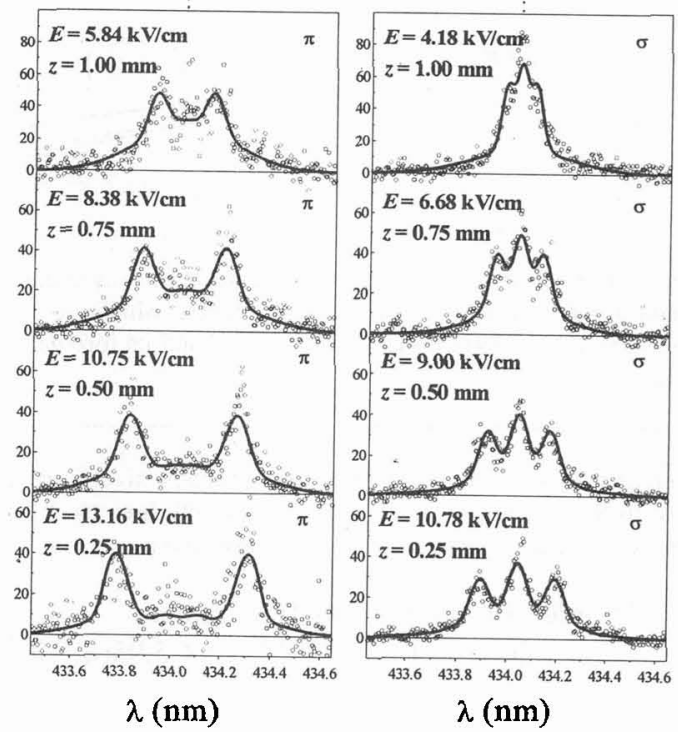

Fig. 5. As in Fig. 4, but for the $\mathrm{H}_{r}$ line.

the cathode fall region of the Grimm GDS, for the local field strength we used an average value determined from $\pi$ and $\sigma$ profiles, see [17]. The obtained data are used to calculate the cathode fall potential $U_{i}=\int_{0}^{L} E d z$, which is compared with the measured cathode-anode potential $U_{0}$. If one takes into account the uncertainties in electric field measurements, one may conclude that the agreement within $\pm 10 \%$ between electric field measurements from the $\mathrm{H}_{\beta}$ and $\mathrm{H}_{\gamma}$ line profiles is satisfactory, see [17]. The agreement between the measured cathode-anode voltage $U_{0}$ and the potential fall derived from the electric field measurements indicates that, for the present experimental conditions, the whole potential applied to the electrodes is consumed in the cathode fall region.

Typical results of electric field axial distribution, obtained by fitting $\pi$ and $\sigma$ profiles of both $\mathrm{H}_{\beta}$ and $\mathrm{H}_{y}$ lines through the cathode fall region, are presented in Fig. 6 . The usual linear fit of the electric field intensity in the CFR is applied and shown in Fig. 6 also.

The origin of two groups of excited hydrogen atoms may be related to the presence of $\mathrm{H}^{+}, \mathrm{H}_{2}^{+}$and $\mathrm{H}_{3}{ }^{+}$ions which are accelerated in the cathode fall region. Striking the cathode, these ions are neutralized, fragmented into atoms and reflected back towards the negative glow region. After the reflection from the cathode, neutrals collide mainly with $\mathrm{H}_{2}$ and excite, see e.g. [16]. We have shown [17] that only two groups of hydrogen atoms, originated from $\mathrm{H}^{+}$and $\mathrm{H}_{3}{ }^{+}$ions, have enough energy to exceed threshold for Balmer lines excitation. "Slow" $H$ atoms, whose average temperatures in. different experimental conditions vary in our case from $3.4-8.2 \mathrm{eV}$ are, most likely, excited in collisions of hydrogen atoms from $\mathrm{H}_{3}{ }^{+}$with $\mathrm{H}_{2}$. On the other hand, the origin of "fast" excited $\mathrm{H}$ atoms, with average temperatures between $80 \mathrm{eV}$ and $190 \mathrm{eV}$, may be related to energetic $\mathrm{H}^{+}$ions only, see [17]. The typical axial distributions of both "slow" and "fast" excited hydrogen atoms temperature are shown in Fig. 7. The temperature distributions show a decrease with the distance from the cathode, which corresponds well 


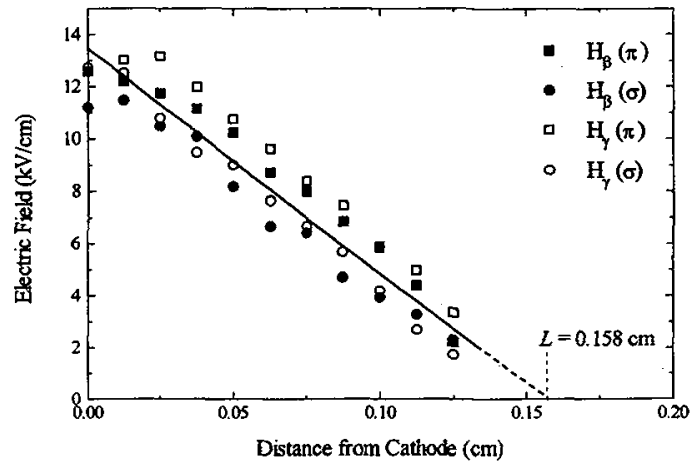

Fig. 6. Electric field distribution in the cathode fall region of the Grimm GDS, operating in pure hydrogen at $228 \mathrm{~Pa}, 30$ $\mathrm{mA}, 920 \mathrm{~V}$. Solid line corresponds to the usual linear fit.

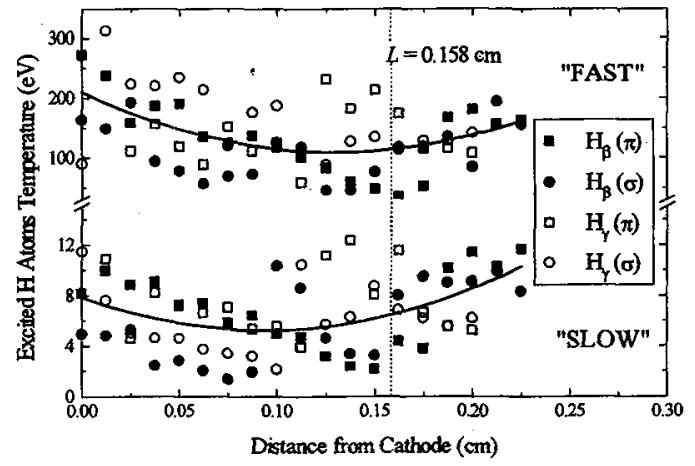

Fig. 7. Axial distribution of "slow" and "fast" excited hydrogen atoms temperature in the cathode fall region of the Grimm GDS, operating in pure hydrogen at $228 \mathrm{~Pa}, 30 \mathrm{~mA}$, $920 \mathrm{~V}$.

with decreasing energy of the reflected atoms. An unexpected rise of the temperature in the negative glow region, see Fig 7 , could be related to the additional collision processes with electrons, whose concentration is rather large $\left(\approx 10^{14} \mathrm{~cm}^{-3}\right.$, see [14]). Here, most likely, one has a superposition of profiles originating from two different excitation processes: one resulting from collisions with heavy particles, and another - collisions of fast neutrals with electrons. Assuming an exponential decrease of the number of reflected energetic atoms due to collisions with the matrix gas, a simple calculation using total cross section data [26] for collisions of neutral hydrogen atoms at $133.4 \mathrm{eV}$ with $\mathrm{H}_{2}$ at $p=228 \mathrm{~Pa}, T_{g}=1000 \mathrm{~K}$ and $L=0.158 \mathrm{~cm}$ shows that about $18 \%$ of the reflected "fast" $\mathrm{H}$ atoms arrive at the negative glow without a collision. In this region they collide with both electrons and matrix gas which results in two groups of excited atoms with different temperatures. Since electrons change the internal atom energy only, the group with larger temperature is the one from atom-electron collisions. Therefore, the resulting profile is broader than in the cathode fall region. Unfortunately, the spectral resolution does not allow us to separate these two profiles, and the results for average temperatures in the negative glow region in Fig. 7 represent some kind of an average value. In addition, the axial intensity distributions of the Balmer lines in hydrogen, see Fig. 8, show a second maximum in the beginning of the negative glow region, which

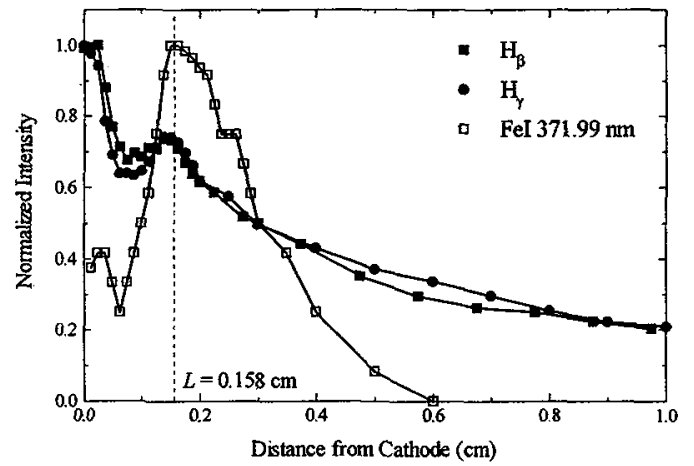

Fig. 8. Normalized axial intensity distributions of the hydrogen $\mathrm{H}_{\beta}$ and $\mathrm{H}_{\gamma}$ Balmer lines and neutral iron line. $L$ is the length of the cathode fall region. Discharge conditions: $\mathrm{H}_{2}$ at $228 \mathrm{~Pa}, 30 \mathrm{~mA}, 920 \mathrm{~V}$. could be another indication of the described fast atom - electron excitation.

From the spatial distribution of the emission of hydrogen and sputtered material, shown in Fig. 8, one can see that the mechanism of hydrogen excitation is different from the mechanism of excitation of the metal atoms. Hydrogen atoms are excited in collisions with $\mathrm{H}_{2}$, while $\mathrm{Fe}$ atoms are excited in collisions with electrons. The intensities of $\mathrm{H}$ lines are the largest close to the cathode surface where the energy and concentration of fast $\mathrm{H}$ atoms are the largest. The intensity of the iron line however, increases gradually from the cathode and this behavior may be related to the gradual increase of electron energy which reaches the optimum for excitation at the beginning of the negative glow region. 

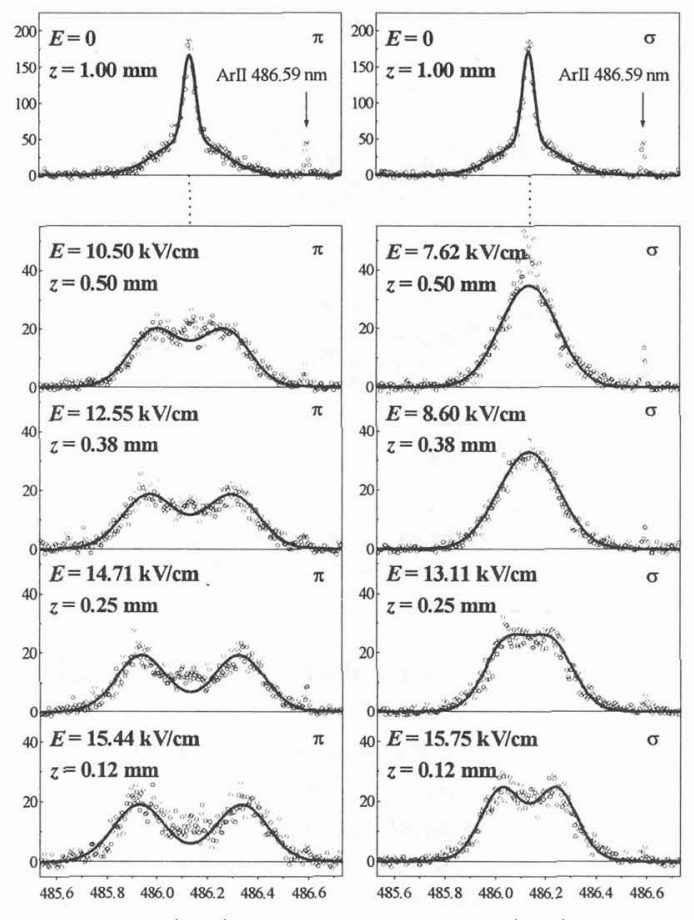

$\lambda(\mathrm{nm})$

$\lambda(\mathrm{nm})$

Fig. 9. As in Fig. 4, but in the $\mathrm{Ar}+3 \% \mathrm{H}_{2}$ mixture. Discharge conditions: $320 \mathrm{~Pa}, 30 \mathrm{~mA}, 820 \mathrm{~V}$.

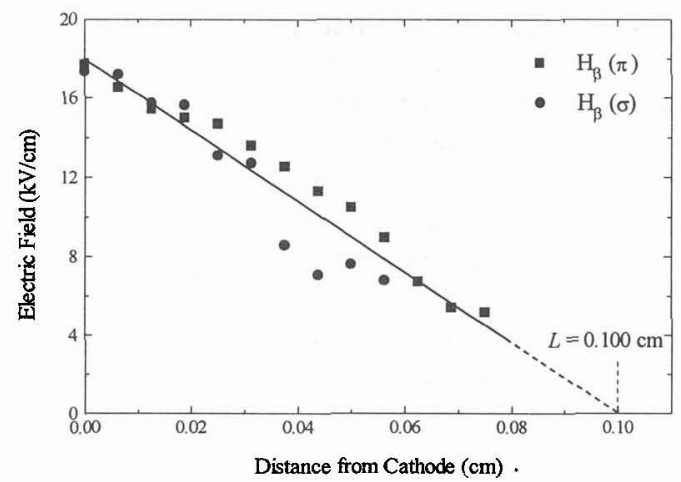

Fig. 10. Electric field distribution in the cathode fall of the Grimm GDS, operating in the $\mathrm{Ar}+3 \% \mathrm{H}_{2}$ mixture at $320 \mathrm{~Pa}$, $30 \mathrm{~mA}, 820 \mathrm{~V}$.

\subsection{Discharge in an argon-hydrogen mixture}

In comparison to pure hydrogen, the discharges in an argon-hydrogen mixture are located closer to the cathode, with smaller lengths of the cathode fall region, so the gradients of electric field in the radial direction are larger. Due to the interference of the Ar I and Ar II lines with the hydrogen $\mathrm{H}_{y}$ line in argon-hydrogen mixture, the electric field measurements are performed using $\pi$ and $\sigma$ profiles of the $\mathrm{H}_{\beta}$ only. Typical examples of the $\mathrm{H}_{\beta}$ Stark profile recordings, best fits and results of electric field measurements are given in Fig. 9. A small central peak superimposed on the $\pi$ and $\sigma$ profiles even close to the cathode arises out of the part of the discharge which is inevitably protruding through the observation slot into the field-free space. Again, differences between results of electric field measurements from $\pi$ and $\sigma$ profiles are detected, and they are also discussed in [17].

Typical results of the electric field distribution in the cathode fall region of the discharge operating in an argon-hydrogen mixture are presented in Fig. 10. As a consequence of the smaller cathode fall region, in comparison with pure hydrogen, see Fig. 6, the electric field intensities at the cathode surface are larger, as well as electric field gradients. Again, experimental electric field distributions are fitted by usual linear fit, presented by the solid line in Fig. 10.

Contrary to pure hydrogen, here one cannot distinguish several groups of excited hydrogen atoms with different velocities in the procedure of $\mathrm{H}_{\beta} \pi$ and $\sigma$ profiles fitting. Namely, only excited $\mathrm{H}$ atoms with energies in the range $32-43 \mathrm{eV}$ exist, see [17]. The overall profiles are smooth, without distinct characteristic peaks induced by "slow" excited atoms, see Fig. 4, which helped us measure the electric field in hydrogen accurately. As a consequence, two typical peaks of $\mathrm{H}_{\beta}(\sigma)$ - profiles can be resolved only in the first few observation positions from the cathode. This certainly affects the accuracy of our measurements.

It is very interesting to note that hydrogen lines in the argon-hydrogen mixture exhibit much stronger wings of the lower part of profile, see Figs. 4 and 9, while the temperatures of excited hydrogen atoms are between those of "slow" and "fast" atoms in pure hydrogen, see [17]. In the gas mixture the role of argon is important for the very efficient production of $\mathrm{H}_{3}{ }^{+}$after the following reactions:

$$
\begin{aligned}
& \mathrm{Ar}^{+}+\mathrm{H}_{2} \rightarrow \mathrm{ArH}^{+}+\mathrm{H}, \\
& \mathrm{ArH}^{+}+\mathrm{H}_{2} \rightarrow \mathrm{H}_{3}^{+}+\mathrm{Ar} .
\end{aligned}
$$

Another two reactions contribute considerably to the increase of $\mathrm{H}_{3}{ }^{+}$concentration in argon-hydrogen mixture: 


$$
\begin{aligned}
& \operatorname{Ar}^{+}\left(3 \mathrm{p}^{52} \mathrm{P}^{0}{ }_{1 / 2}\right)+\mathrm{H}_{2} \rightarrow \mathrm{Ar}+\mathrm{H}_{2}{ }^{+}, \\
& \mathrm{H}_{2}^{+}+\mathrm{H}_{2} \rightarrow \mathrm{H}_{3}^{+}+\mathrm{H},
\end{aligned}
$$

see [16]. A large concentration of $\mathrm{H}_{3}{ }^{+}$ions increases the intensities of the hydrogen line wings. Therefore, $\mathrm{H}_{3}{ }^{+}$is now the dominating hydrogen ion and backscattered $\mathrm{H}$ atoms from the cathode originate mainly from this ion. In comparison with the pure hydrogen discharge, where "fast" $H$ atoms originate from reflection of $\mathrm{H}^{+}$, and "slow" from reflection of $\mathrm{H}_{3}{ }^{+}$ions, here we have excited atoms formed in reflection of $\mathrm{H}_{3}{ }^{+}$ions only. However $\mathrm{H}_{3}{ }^{+}$ions gain more energy in the cathode fall region due to the higher transparency of argon (mass ratio 3:40) than the hydrogen matrix (3:2). Typical axial distribution of the excited hydrogen atoms temperature in the argon-hydrogen mixture discharge is shown in Fig. 11.

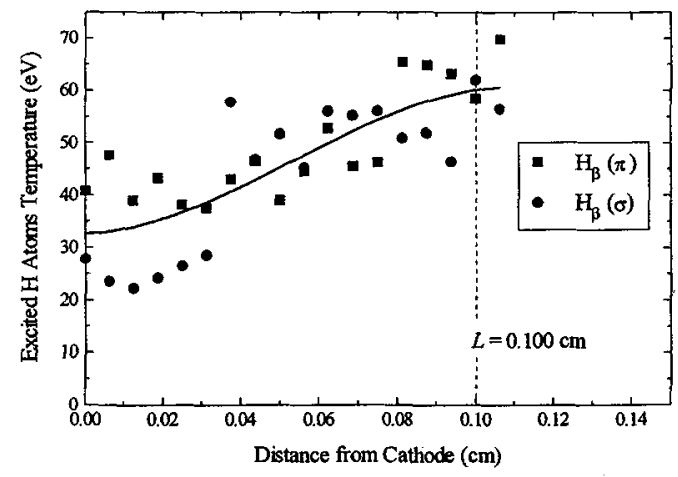

Fig. 11. Axial distribution of the excited hydrogen atoms temperature in the cathode fall region of the Grimm GDS, operating in the argon-hydrogen mixture at $320 \mathrm{~Pa}, 30 \mathrm{~mA}$, $820 \mathrm{~V}$.

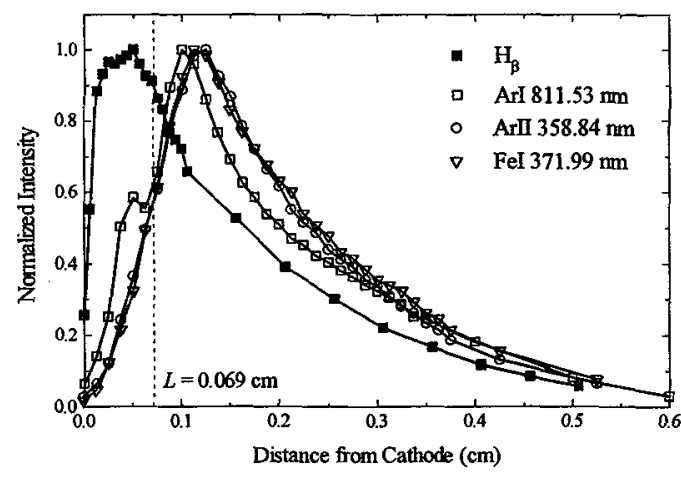

Fig. 12. Normalized axial line intensity distributions of the hydrogen $\mathrm{H}_{\beta}$, neutral and ionized argon, and neutral iron line. $L$ is the length of the cathode fall region. Discharge conditions: $\mathrm{Ar}+3 \% \mathrm{H}_{2}$ at $320 \mathrm{~Pa}, 20 \mathrm{~mA}, 490 \mathrm{~V}$.

From Fig. 11 one can see that the effect of the excited hydrogen atoms temperature increasing towards the negative glow region is even more pronounced in the argon-hydrogen mixture than in pure hydrogen, see Fig. 7. The similar explanation could be drawn: in addition to the excitation of back-reflected hydrogen atoms by collisions with matrix gas, they are excited in collisions with electrons. Once again, assuming an exponential decrease of the number of reflected energetic atoms due to collisions with matrix gas, a simple calculation using total cross section data [27] for collisions of $\mathrm{H}$ atoms at $75 \mathrm{eV}$ with argon at a pressure of $320 \mathrm{~Pa}$ and gas temperature $\left(T_{g}\right)$ of $1000 \mathrm{~K}$, shows that, for a cathode fall length of $0.1 \mathrm{~cm}$, as many as $66 \%$ of the reflected neutrals reach the negative glow region without any collision. Their higher concentration in comparison with pure hydrogen, makes this effect more pronounced in the argonhydrogen discharge.

The axial distributions of the Ar I, Ar II, Fe I and $\mathrm{H}_{\beta}$ line intensities are given in Fig. 12. Again the maximum of intensity of the $\mathrm{H}_{\beta}$ line is close to the cathode surface. However, contrary to pure hydrogen, where the maximum of intensity is at the cathode surface, see Fig. 8, steep rise of the $\mathrm{H}_{\beta}$ intensity in argon-hydrogen mixture is detected. This difference may be explained by a more efficient collisional energy transfer between reflected energetic hydrogen atoms and $\mathrm{H}_{2}$ molecules (mass ratio 1:2) than in case of argon (1:40). The second maximum in the $\mathrm{H}_{\beta}$ line intensity axial distribution, clearly observed in pure hydrogen, see Fig. 8, is partially covered here by the first maximum, which is slightly shifted away from the cathode surface towards the negative glow, see Fig. 12.

\subsection{Discharge in pure helium and in a helium-hydrogen mixture}

To obtain local electric field strengths in pure helium and in helium-hydrogen mixture discharges, we used polarization-dependent Stark splitting and shifting of helium lines, as described in Section 3.2. As an illustration of the influence of the electric field strength on the wavelength position of the He I $492.1 \mathrm{~nm}$ and He $1447.1 \mathrm{~nm}$ allowed and their forbidden lines, the typical $\pi$ polarized spectra, recorded at several 

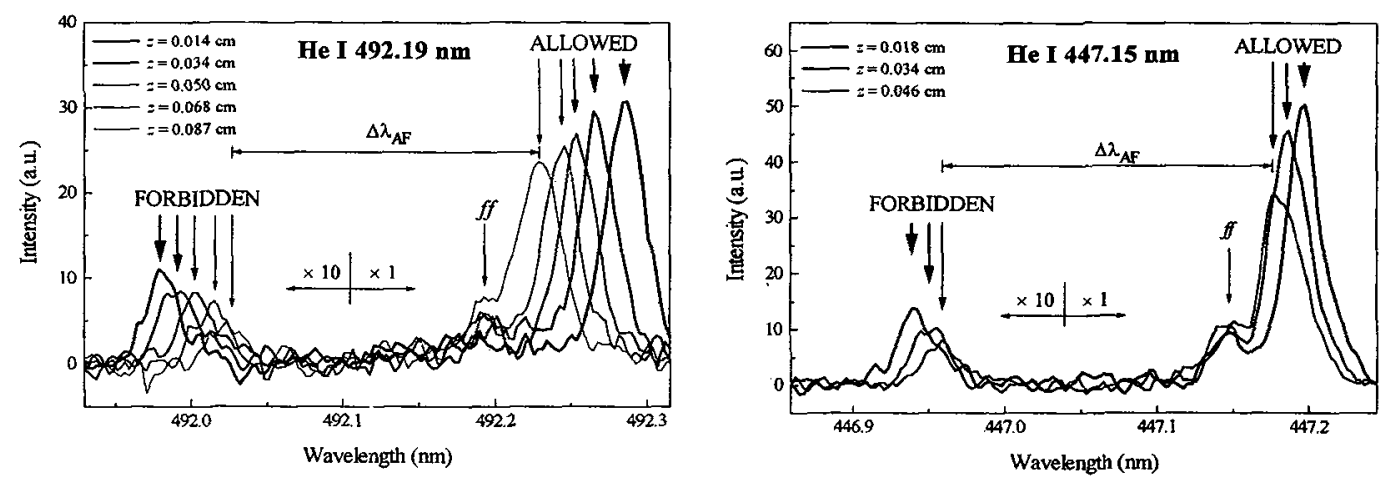

Fig. 13. Typical $\pi$ polarized spectra of the He I $492.19 \mathrm{~nm}$ and He I $447.1 \mathrm{~nm}$ lines and their forbidden counterparts, recorded at several distances from the cathode surface of the Grimm GDS operating in pure helium. Discharge parameters: $128 \mathrm{~Pa}, 20$ $\mathrm{mA}, 1500 \mathrm{~V}$. Unshifted peaks denoted by $f f$ (field-free) are emitted from the plasma protruding through the observation slot. The forbidden lines are recorded with 10 times larger detection sensitivity.

distances from the cathode of the Grimm GDS, are given in Fig. 13. The unshifted maxima in these figures originate from the discharge protruding through the observation slot into the field-free space. To facilitate mutual agreement of the results obtained from three He I lines for the same experimental conditions, we have measured the electric field distribution in the CFR of our glow discharge and the results are given in Fig. 14.

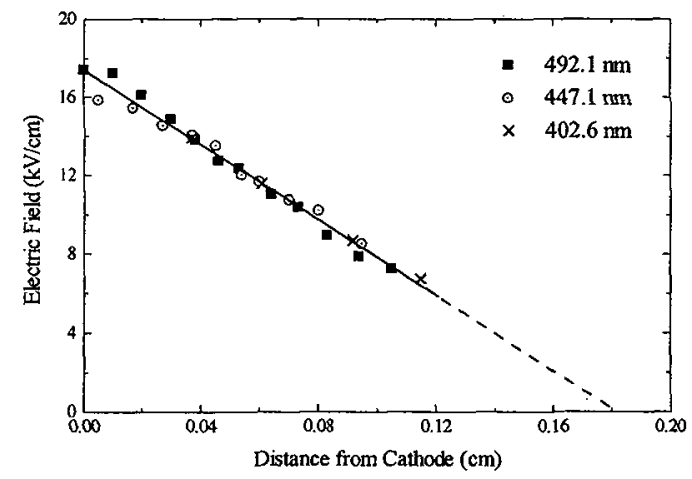

Fig. 14. The axial distribution of the electric field determined from $\Delta \lambda_{\mathrm{AF}}$ for all three He I lines in the CFR of the Grimm GDS. Experimental conditions as in Fig. 13.

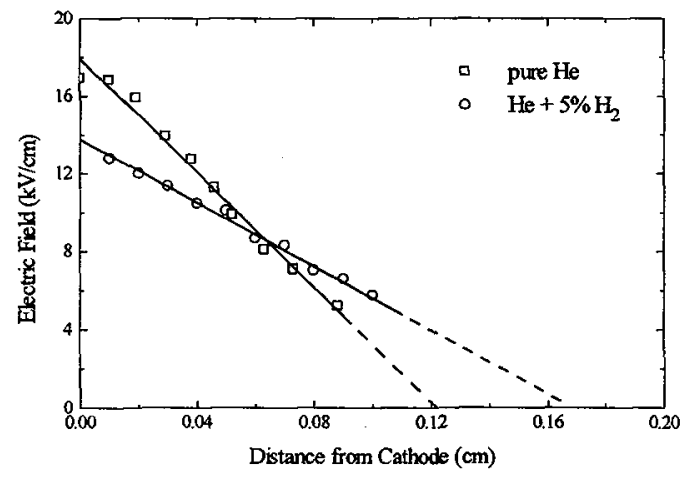

Fig. 15. The axial distribution of the electric field strength in pure helium and in the helium-hydrogen mixture for the same discharge conditions: $202 \mathrm{~Pa}, 20 \mathrm{~mA}, 1050 \mathrm{~V}$.

To compare the results in pure helium and in the helium-hydrogen mixture, we had to establish the same discharge conditions (pressure, current and voltage) in both gases. This is achieved by changing the gas flow ratio in two vacuuming branches of our Grimm GDS lamp, see [19]. The typical results of electric field axial distribution, measured using the He I $492.19 \mathrm{~nm}$ line, are given in Fig. 15. It is important to notice that even a small amount $(5 \%)$ of hydrogen decreases the electric field intensity at the cathode surface by $\approx 25 \%$ and extends the length of the CFR by $\approx 30 \%$.

In pure helium, the measurements are performed using the $\mathrm{He} I 492.19 \mathrm{~nm}$ line, for a $20 \mathrm{~mA}$ discharge current at three different pressures $(128,145$ and $202 \mathrm{~Pa})$. It is interesting that for all pressures the maximum electric field at the cathode surface remains the same, and only the length of the CFR changes, see Fig. 16. This result suggests that, in this range of discharge conditions, the electric field strength at the cathode surface does not depend upon the applied voltage and the pressure. To check our experimental results of the local electric field intensity, the cathode fall potential $U_{1}=\int_{0}^{t} E d z$ is calculated and compared with the measured anode-cathode voltage $U_{0}$. As in our above described measurements in pure hydrogen and the argon-hydrogen mixture [17], $U_{i}$ is approximately equal to the total anode-cathode 


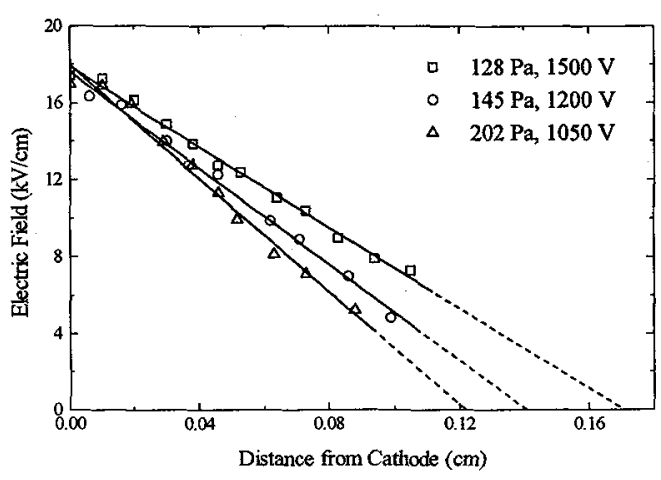

Fig. 16. The results of the electric field axial distribution measurements in pure helium at three different pressures.

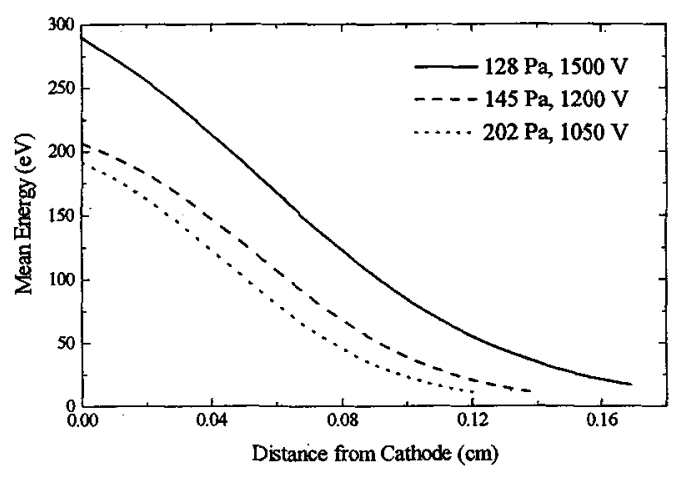

Fig. 17. Spatial distributions of $\mathrm{He}^{+}$ion mean energies in the CFR of the Grimm GDS in pure helium.

voltage $U_{0}$, indicating that most of the applied voltage is consumed in the CFR of the Grimm GDS. The obtained axial distributions of the electric field strength in pure helium are used to calculate the mean free paths of the $\mathrm{He}^{+}$ions generated in symmetrical charge exchange processes and their mean energy distributions in the CFR. For this purpose, the theory of Wronski [28] is applied, the basics of which are given in detail in $[17,19]$. The results of these calculations, i.e. spatial distributions of the $\mathrm{He}^{+}$ion mean energies for three investigated pressures of pure helium, are given in Fig. 17. It is interesting to notice that the maximum mean ion energy at the cathode surface $(190-290 \mathrm{eV})$ represents approximately $20 \%$ of the anode-cathode voltage, see Fig 17 . The same correlation is found between the mean free path and the length of cathode fall region, see [19].

\section{CONCLUSIONS}

In this paper we presented results of the experimental study of the cathode fall region of a Grimm-type glow discharge in pure hydrogen, an argon-hydrogen mixture, pure helium and a helium-hydrogen mixture. First, the details of the experimental setup are given, with the emphasis on the improvements in the helium-based experiment. This is followed by the short reviews of the theoretical basis of our measurements: Stark splitting of the hydrogen Balmer $\mathrm{H}_{\beta}$ and $\mathrm{H}_{\gamma}$ lines in an external electric field, and Stark splitting and shifting of the neutral helium $492.19 \mathrm{~nm}, 447.15 \mathrm{~nm}$ and $406.62 \mathrm{~nm}$ lines with respect to their forbidden counterparts.

In the pure hydrogen discharge, the results of electric field strength axial distributions obtained from the $\mathrm{H}_{\beta}$ and $\mathrm{H}_{\gamma}$ lines show good mutual agreement, see Figs. $4-6$. Doppler spectroscopy of the same Balmer lines showed that at least two groups of excited hydrogen atoms with considerably different velocities exist in the discharge. The origin of "slow" neutrals, with average energies in the range $3.4-8.2$ $\mathrm{eV}$ is related to the existence of $\mathrm{H}_{3}{ }^{+}$ions which are accelerated in the CFR, neutralized and fragmented into atoms at the cathode surface, and back-reflected towards the negative glow region. The other group of "fast" hydrogen atoms, with average energies ranging between 80 and $190 \mathrm{eV}$, most likely originate from the neutralization and reflection of energetic $\mathrm{H}^{+}$ions. These back-reflected atoms, on their way to the negative glow region, collide mostly with $\mathrm{H}_{2}$ and excite. Approaching the negative glow region, the increase of both "slow" and "fast" hydrogen atoms temperature is detected, see Fig. 7, and related to the additional excitation in collisions with electrons.

The discharge in the argon-hydrogen mixture is located closer to the cathode and, therefore, higher electric fields at the cathode surface as well as higher field gradients are detected, see Fig 9. Due to the shorter CFR and the absence of "slow" hydrogen atoms, the electric field measurements are less accurate, see Fig 10. Namely, in the argon-hydrogen mixture only one group of excited hydrogen atoms is detected, with average energies in the range $32-43 \mathrm{eV}$. Their origin is related to the dominant role of $\mathrm{H}_{3}{ }^{+}$ions in the discharge. The temperature increase towards the negative glow region is even more pronounced than in pure hydrogen, see Fig. 11, due to the higher number of energetic neutrals which reach the negative 
glow without any collision. In both gases, the axial intensity distributions of hydrogen Balmer lines and lines of sputtered material and matrix gas, see Figs. 8 and 12, confirm the different excitation mechanism of hydrogen lines in comparison with all others. Balmer lines are excited in collisions with heavy particles, while the excitation of lines of other species results from the collisions with electrons.

Finally, in pure helium and helium-hydrogen discharges, the method of electric field measurements using Stark splitting and shifting of neutral helium allowed and forbidden lines is demonstrated. Good mutual agreement between electric field measurements from three helium lines is obtained, see Fig 14. It is detected that even a small amount of hydrogen considerably changes $(\approx 25 \%)$ the electric field intensity at the cathode surface and the length of CFR, see Fig. 15. In pure helium, for all three investigated pressures, the electric field at the cathode surface remains constant (around $17 \mathrm{kV} / \mathrm{cm}$ ), and the length of CFR changes only, see Fig. 16. By applying the theory of Wronski [28], the spatial distributions of $\mathrm{He}^{+}$ ion mean energies in the CFR of pure helium discharge are obtained, see Fig. 17. At the cathode surface, maximum mean ion energies range from $190-290 \mathrm{eV}$ at the present discharge conditions, see Fig. 17.

\section{Acknowledgments}

The loan of a $4 \mathrm{~m}$ spectrometer from the Paris Observatory - Meudon is gratefully acknowledged. This work is supported by the Ministry of Science and Technology of the Republic of Serbia.

\section{References}

[1] Grimm W., Spectrochim. Acta Part B 23 (1968) 443-454.

[2] Dogan M., Laqua K., Maßmann H., Spectrochim. Acta Part B 26 (1971) 631-649.

[3] Dogan M., Laqua K., Maßmann H., Spectrochim. Acta Part B 27 (1972) 65-88.

[4] Broekaert J.A.C., J. Anal. At. Spectrom. 2 (1987) 537-545.

[5] Berneron R., Spectrochim. Acta Part B 33 (1978) 665-673.

[6] Koch K.H., Kretschmer M., Grunenberg D., Microchim. Acta II (1985) 1-25.

[7] Gough D.S., Anal. Chem. 48 (1976) 1926-1931.

[8] Ohls K., Fresenius' Z. Anal. Chem. 327 (1987) 111-118.

[9] Harrison W.W., Bentz B.L., Prog. Anal. Spectrosc. 11 (1988) 53-59.

[10] Parker M., Marcus R.K., Appl. Spectrosc. 48 (1994) 623-631.

[11] Boumans P.W.J.M., Spectrochim. Acta Part B 46 (1991) 711-739.

[12] Broekaert J.A.C., Appl. Spectrosc. 49 (1995) 12A-19A.

[13] Boumans P.W.J.M., Broekaert J.A.C., Marcus R.K., Eds. Spectrochim. Acta Part B 46 (2) (1991) 111-313; 46 (4) (1991) 457-546.

[14] Kuraica M., Konjević N., Platiša M., Pantelić D., Spectrochim. Acta Part B 47 (1992) 1173-1186.

[15] Kuraica M., Konjević N., Phys. Rev. A 46 (1992) 4479-4482.

[16] Kuraica M., Konjevic N., Phys. Scripta 50 (1994) 487-492.

[17] Videnovic I.R., Konjević N., Kuraica M.M., Spectrochim. Acta Part B 51 (1996) 1707-1731.

[18] Kuraica M.M., Konjević N., Appl. Phys. Lett. 70 (1997) 1521-1523.

[19] Kuraica M.M., Konjević N., Videnović I.R., Spectrochim. Acta Part B 52 (1997) 745-753.

[20] Ferreira N.P., Human H.G.C., Butler L.R.P., Spectrochim. Acta Part B 35 (1980) 287-295.

[21] Condon E.U., Shortley G.H., The Theory of Atomic Spectra, (University Press, Cambridge, 1977).

[22] Ryde N., Atoms and Molecules in Electric Fields,

(Almquist \& Wiksell International, Stockholm, 1976).

[23] Bogaerts A., van Straaten M., Gijbels R., Spectrochim. Acta Part B 50 (1995) 179-196.

[24] Foster J.S., Proc. R. Soc. London A 117 (1927) 137-163.

[25] Martin W.C., J. Phys. Chem. Ref. Data 2 (1973) 257-265.

[26] Phelps A.V., J. Phys. Chem. Ref. Data 19 (1990) 653-675.

[27] Phelps A.V., J. Phys. Chem. Ref. Data 21 (1992) 883-897.

[28] Wronski Z., Vacuum 40 (1990) 387-394. 